\title{
Cerebrospinal fluid analysis and the determination of oligoclonal bands
}

\author{
Matteo Gastaldi ${ }^{1}$ Elisabetta Zardini ${ }^{1} \cdot$ Rosaria Leante $^{2} \cdot$ Maddalena Ruggieri $^{2}$. \\ Gianna Costa ${ }^{3}$ - Eleonora Cocco ${ }^{3}$ - Giovanna De Luca ${ }^{4}$ Ivana Cataldo ${ }^{4}$. \\ Tiziana Biagioli $^{5}$ - Clara Ballerini $^{6} \cdot$ Massimiliano Castellazzi $^{7} \cdot$ Enrico Fainardi $^{6}$. \\ Paola Pettini $^{8}$ - Mauro Zaffaroni ${ }^{8}$ - Debora Giunti ${ }^{9}$ - Elisabetta Capello ${ }^{9}$. \\ Gaetano Bernardi ${ }^{10}$ - Emilio Ciusani ${ }^{10}$. Claudia Giannotta ${ }^{11}$. \\ Eduardo Nobile-Orazio ${ }^{11}$ Elena Bazzigaluppi ${ }^{12}$ - Gabriella Passerini ${ }^{12}$. \\ Roberta Bedin ${ }^{13}$ - Patrizia Sola ${ }^{13}$ - Rinaldo Brivio ${ }^{14}$. Guido Cavaletti ${ }^{15}$. \\ Arianna Sala ${ }^{16}$ • Antonio Bertolotto ${ }^{16}$. Gaetano Desina ${ }^{17}$ - Maurizio A. Leone ${ }^{17}$. \\ Sara Mariotto ${ }^{18}$ - Sergio Ferrari ${ }^{18}$ - Andreina Paternoster ${ }^{19} \cdot$ Davide Giavarina $^{19}$. \\ Francesco Lolli ${ }^{6}$ Diego Franciotta ${ }^{20}$
}

(C) Springer-Verlag Italia S.r.1. 2017

\begin{abstract}
This document presents the guidelines for the cerebrospinal fluid (CSF) analysis and the determination of oligoclonal bands (OCBs) as pivotal tests in neuroin flammatory pathologies of the central nervous system. The guidelines have been developed following a consensus process built on questionnaire-based surveys, internet contacts, and discussions at workshops of the sponsoring
\end{abstract}

Diego Franciotta

diego.franciotta@mondino.it

1 C. Mondino National Neurological Institute, University of Pavia, Pavia, Italy

2 AOU Policlinico di Bari, Bari, Italy

3 Ospedale R. Binaghi, Cagliari, Italy

4 Policlinico SS. Annunziata, Chieti, Italy

5 AOU Careggi, Florence, Italy

6 Department of Biomedical, Experimental and Clinical Sciences, University of Florence, Florence, Italy

7 Department of Biomedical and Specialty Surgical Sciences, University of Ferrara, Ferrara, Italy

8 Ospedale di Gallarate ASST Valle Olona, Gallarate, Italy

9 Ospedale Policlinico San Martino IRCCS, University of Genova, Genoa, Italy

10 C. Besta Neurological Institute, Milan, Italy
Italian Association of Neuroimmunology (AINI) congresses. Essential clinical information on the pathologies in which the CSF analysis is indicated, and, particularly, on those characterized by the presence of OCBs in the intrathecal compartment, indications and limits of CSF analysis and OCB determination, instructions for result interpretation, and agreed laboratory protocols (Appendix) are
11 Humanitas Clinical Institute, Milan University, Milan, Italy

12 Ospedale San Raffaele, Milan, Italy

13 Ospedale Civile Azienda Ospedaliero-Universitaria di Modena, Modena, Italy

14 ASST Monza, Monza, Italy

15 Expersimental Neurology Unit, University of Milano-Bicocca, Monza, Italy

16 AUO S. Luigi, Orbassano, Italy

17 IRCCS “Casa Sollievo della Sofferenza”, San Giovanni Rotondo, Italy

18 Department of Neuroscience, Biomedicine and Movement, University of Verona, Verona, Italy

19 Ospedale S. Bortolo, Vicenza, Italy

20 Laboratory of Neuroimmunology, C. Mondino National Neurological Institute, Via Mondino 2, 27100 Pavia, Italy 
reported for the communicative community of neurologists and clinical pathologists.

Keywords Intrathecal IgG synthesis · Isoelectric focusing . Laboratory diagnostics $\cdot$ Multiple sclerosis .

Neuroimmunology

\section{Introduction}

Cerebrospinal fluid (CSF) examination has produced and keeps producing a large amount of data without substantial standardization of procedures and techniques. The European consensus on the CSF in the diagnosis of multiple sclerosis (MS) [1] did not fully deal with the issue of procedure and method standardization, as a subsequent publication highlighted the need of identifying shared technical and interpretative guidelines [2].

Since 2000, when the Italian Association of Neuroimmunology (AINI) started promoting and managing the process of standardization of methods and production of guidelines, the judgment on the CSF analysis state of the art moved from "nearly sufficient," as reported by a 1995 survey of the Italian Society of Clinical Biochemistry [3], to "very good," as extrapolated by the data of AINI external quality control schemes between 2000 and 2014. In summary, AINI centers (a) have applied the main methodological and procedural principles of the 1994 consensus [1]; (b) currently use standardized and certified methods for the determinations of albumin and $\mathrm{IgG}$, which allow the evaluation of blood-CSF barrier damage and intrathecal IgG production, respectively; and (c) exploit the improvements of automation in isoelectric focusing (IEF) techniques, used by a growing number of centers (nothwithstanding that semi-automated instruments for IEF use mini-gels that might perform suboptimally [4], the AINI controls show that the quality of IEF runs has been improving over years).

The area of CSF biomarkers needs standardization too. For example, in MS, the lack of standardization is one of the main reasons that prevent the entry of new claimed-to-be-effective biomarkers into routine diagnostics. Other reasons include cutoff values for discriminating between patients and controls first optimized and then applied to the same cohorts, thus artificially increasing test sensitivity and specificity, and evaluations of single biomarkers instead of a set of biomarkers, which could allow the real diagnostic power of each of them. Accordingly, a recent and comprehensive review maintains that no new MS biomarker have entered routine diagnostics so far, although the CSF IgM oligoclonal band (OCB) test is the most promising one [5]. Again, the test has never undergone a thorough process of validation and standardization.

\section{Clinical and laboratory aspects}

The evaluation of diagnostic performance of CSF tests is complex for the following reasons: lumbar puncture is usually not repeated, CSF volumes obtained are limited, CSF samples from healthy controls are not easily available, and CSF abnormalities are disease-specific in very few cases only. Therefore, in addition to low levels of standardization, sensitivity and specificity of CSF tests often show between-study moderate-high ranges of variation. All these considerations should dictate rational requests for CSF analysis, which should follow thorough evaluations of clinical settings and disease prevalences (Bayes' theorem). The main pathological conditions for which the lumbar puncture is indicated are listed in Table 1.

Urgent CSF analysis, which includes cell count, glucose, and albumin determination in serum and CSF (CSF total protein as an alternative), is mandatory in suspected infectious meningitis/meningoencephalitis.

Serum and CSF albumin determinations, with calculation of albumin quotient, allow the most accurate measurement of the degree of blood-CSF barrier permeability ("blood-CSF barrier damage"-which does not correspond to "bloodbrain barrier damage"), and should replace CSF total proteins in disorders characterized by intrathecal IgG production at least [1].

IEF full standardization is inherently impossible due to imponderable factors (gel conductivity, electrodes soaking, lot-to-lot differences for ampholytes, electro endosmosis phenomena, environment temperature/degree of humidity).

Table 1 Indications for lumbar puncture for diagnostic purposes

Infectious meningitis and meningoencephalitis

Multiple sclerosis

Autoimmune encephalitis

Paraneoplastic neurological syndromes

Acute disseminated encephalomyelitis (ADEM)

Neuromyelitis optica spectrum disorders (NMOSD)

Dysimmune polyradicoloneuritis

Cranial mono/multinevritis

Meningeal carcinomatosis

Neurological involvement in inflammatory/autoimmune systemic diseases

Benign intracranial hypertension

Normal pressure hydrocephalus

Subarachnoid hemorrhage

Creutzfeldt-Jacob disease

Alzheimer disease 


\section{Autoimmune encephalitis}

Testing for autoantibodies to surface membrane/synaptic neuronal antigens is recommended for the differential diagnosis of encephalitis $[6,7]$. Testing CSF samples is mandatory for the detection of anti- $N$-methyl-D-aspartate receptor (NMDAR) antibodies, since false negative results have been reported in $14 \%$ of the cases testing serum samples only [8]. Preliminary findings suggest that repeated CSF titrations of these antibodies can provide information on prognosis and response to therapy [8].

\section{Multiple sclerosis and oligoclonal bands}

The 1994 consensus report contains seminal and still valid principles for the CSF analysis ([1]; "Appendix"). One fundamental recommendation refers to the use of "immunofixation after the isoelectric focusing step" for OCB detection. In 2005, the message was reinforced by a joint North AmericanEuropean consensus [9]. A "five-pattern scheme" for IEFimmunofixation test interpretation represents another essential contribution to the OCB pattern interpretation [1]. Minor modifications to this scheme have been suggested ("Appendix").

The "2010 revised McDonald criteria" for MS diagnosis downplayed the diagnostic role of CSF testing, considered as supportive criterion for the diagnosis of primary-progressive MS only [10]. The criterion entails equivalence between CSF OCBs and abnormal IgG Index values, although IgG Index is less sensitive than OCBs for the intrathecal IgG synthesis detection, and at risk of false positive results in the presence of moderate/severe blood-CSF barrier damage [11]. The substantial exclusion of CSF analysis from MS diagnostic criteria has generated conflicting opinions (reviewed in [12]). Notably, however, in patients with clinically isolated syndrome (CIS), CSF OCBs help define the risk of MS conversion with $91 \%$ sensitivity and $94 \%$ specificity [13], with greater accuracy vs the criterion of dissemination in space on brain MRI (70 vs 58\%) and improvement of global diagnostic accuracy [14]. Most importantly, the exclusion of alternative diagnosis, which requires CSF analysis, is also fundamental in CIS patients [15] and in general in patients with suspected CNS inflammatory diseases.

On the prognostic side, the revived association between absence of CSF OCB and benign MS prognosis [16], has not been confirmed [17]. Moreover, some studies suggested that CSF OCBs of the IgM isotype associate with (a) aggressive MS forms [18], (b) better responses to immunotherapies [19], (c) lower risks of developing progressive multifocal leukoencephalopathy in patients on natalizumab [20], and (d) higher probability of reaching EDSS scores of 3.0/4.0 10 years after onset [21]. The data are waiting for further confirmation and wider consensus before IgM OCB test enters routine clinico-laboratory practice [22].

\section{Neuromyelitis optica spectrum disorders}

CSF is less sensitive than serum for the detection of AQP4IgG [23]. Testing CSF samples is restricted to seronegative NMOSD patients comorbid for other autoimmune diseases, as these systemic diseases possibly associate with circulating autoantibodies that can interfere with anti-AQP-4 antibody detection [24].

\section{Creutzfeldt-Jacob disease}

Western blot for CSF protein 14.3.3 determination is the reference test for the diagnosis of Creutzfeldt-Jacob disease [25]. Tau protein ELISA, which allows quantitative measurements, has high specificity and specificity $(>90 \%)$ [26], and could complement or replace the semiquantitative Western blot technique for protein 14.3.3 determination.

\section{Alzheimer disease}

A consensus paper by the "Alzheimer's Biomarkers Standardization Initiative" on one hand insisted on the need for standardization of biomarkers in $\mathrm{AD}$ and, on the other hand, recommended the routine practice of measuring the CSF concentrations of protein tau, hyperphosphorylated protein tau, and amyloid beta ${ }_{1-42}$ in patients with early onset dementia, prodrome of the disease, and atypical forms of AD [27]. Today, a good level of standardization has been reached thanks also to international quality control schemes. The typical modifications of the three biomarkers allow the diagnosis of $\mathrm{AD}$ in the initial phases, whereas normal values exclude the disease [27]. Regrettably, how an early diagnosis of $\mathrm{AD}$ may be relevant in prognostic and therapeutic terms is still rather unclear.

\section{Amyotrophic lateral sclerosis}

CSF neurofilaments are increasingly emerging as important biomarkers of axonal damage. Their determination is useful for the differential diagnosis of motor neuron diseases, and therefore, they should be included in the diagnostic workup of the disease [28].

\section{Inflammatory neuropathies}

Increased values of CSF total proteins or albulmin quotient with normal CSF cell counts (albumino-cytologic dissociation) are often found in patients with GuillainBarré syndrome [29] or chronic inflammatory demyelinating polyradiculoneuropathy, in which they are considered one of the supportive criteria for the diagnosis [30]. 


\section{Compliance with ethical standards}

Conflict of interest The authors declare that they have no conflict of interest.

\section{Appendix}

\subsection{Preanalytical procedures}

1.1 Lumbar puncture is usually performed in the morning on fasting patients.

1.2 CSF should be collected in sterile siliconized-glass/ polypropylene tubes (glass tubes favor monocyte adhesion).

1.3 Volumes of lumbar-drawn CSF should be standardized $(4-5 \mathrm{~mL})$ and preferably collected in a single tube, because of the following:

i) There is a protein concentration gradient between the first and the last milliliters of drawn CSF [31]; standardized CSF volumes allow accurate comparisons on follow-up analysis and in research settings.

ii) CSF cell collection is maximized.

1.4 When microbiological tests are required, top priority is given to sterility and one additional single CSF aliquot should be collected.

1.5 Use of atraumatic needles (e.g., Sprotte ${ }^{\circledR} 25 \mathrm{~S}$ ) can minimize the frequency of post-lumbar puncture headache and allow safe collection of up to $20 \mathrm{~mL}$ of CSF [32], useful for cell studies.

1.6 A blood sample is always taken simultaneously with the lumbar puncture.

1.7 Paired blood and CSF samples should be sent to the laboratory as soon as possible (within $2 \mathrm{~h}$ after collection).

1.8 Procedures for sending samples to external laboratories (microbiology, pathology, etc.) should be agreed in advance.

1.9 Blood samples should not be hemolyzed or lipemic.

1.10 In cases of traumatic lumbar puncture or suspected subarachnoid hemorrhage:

i) Collect CSF samples in three tubes.

ii) Use the least contaminated tube for analysis.

iii) A not-decreasing-into-three-tube contamination could indicate subarachnoid hemorrhage, without excluding traumatic lumbar puncture.

1.11 Blood-contaminated CSF samples:

i) If obtaining a non-contaminated sample is impossible, blood-contaminated CSF samples are analyzed nonetheless (if there are no clots and they are clear and colorless after centrifugation).

ii) Correcting CSF parameters taking into account erythrocyte numbers is inaccurate. No abnormal test results argue for a "normal CSF sample," whereas abnormalities require critical interpretations in relation to other paraclinical findings and each clinical case.

iii) Samples should be excluded from research protocols.

\subsection{Analytical procedures}

2.1 Visual assessment and spectrophotometric analysis

2.1.1 Assess appearance and color before and after centrifugation at $1500-3000 \mathrm{rpm}$ (about $500 \times \mathrm{g}$ ) for $10 \mathrm{~min}$.

2.1.2 For collecting cell pellets, centrifuge CSF samples with swinging-bucket centrifuges.

2.1.3 Qualitative scales should be used for appearance (e.g., "crystal clear," "cloudy," "turbid") and color ("colorless," "xantocromic," "erythrocromic").

2.1.4 Spectrophotometry should be performed only in suspected subarachnoid hemorrhage or to demonstrate previous hemorrhages [33]. A normal brain CT scan does not rule out subarachnoid hemorrhage.

i) Use centrifuged CSF samples.

ii) Spectrophotometric peaks of 415 and $460 \mathrm{~nm}$ indicate high total protein levels (usually due to blood-CSF-barrier damage).

iii) Spectrophotometric peaks of hemoglobin $(\mathrm{Hb})$ degradation products: the early metabolites (4-8 days) oxidized- and meta-Hb: 415,540 , and $580 \mathrm{~nm}$ indicate recent, and the late metabolites (15-20 days) bilirubin and bilirubin compounds: 350,400 and $460 \mathrm{~nm}$, late subarachnoid hemorrhage.

2.2 CSF cells

2.2.1 CSF cell analysis should be performed within $2 \mathrm{~h}$ after CSF collection [34].

2.2.2 Use at least $100 \mu \mathrm{L}$ of CSF sample for cell count, after gentle agitation of the tube.

2.2.3 Allowed counting chambers: Fuchs-Rosenthal, Bürker, and Nageotte.

2.2.4 Turk's solution (1:1 dilution) helps lyse erythrocytes and counterstain CSF cells.

2.2.5 CSF cell identification: after the first CSF sample centrifugation, the resulting cell pellets are processed with cytocentrifuges (cytospins) or sedimentation chambers (search for tumor cells should pertain to anatomical pathologists).

2.2.6 Fix cell pellets with $10 \%$ formaldehyde $(50 \mu \mathrm{L})$ for later analysis.

2.2.7 Stain CSF cells with May-Grünwald-Giemsa (differential count). 
2.2.8 Morphologic CSF cell analysis is mandatory in suspected meningeal carcinomatosis even in the absence of pleocytosis.

2.2.9 Reporting leukocyte differential counts is optional, unless required by the clinical suspicion.

2.2.10 Flow cytometers, useful for counting and phenotyping CSF cells, are indispensable for assessing B cell clonality in suspected CNS lymphomas.

2.2.11 In blood-contaminated CSF samples, the number of erythrocytes adds no information on the degree of blood-derived cell contamination.

2.3 Biochemical analysis

2.3.1 Perform biochemical analysis on supernatants of centrifuged CSF samples.

2.3.2 Test paired serum and CSF samples.

2.3.3 Basic tests (glucose, albumin, IgG)

2.3.3.1 The percentage of serum-to-CSF glucose transfer (CSF glucose/serum glucose $\times 100$ ) should replace glycorrachia (CSF glucose levels physiologically depend on serum glucose levels).

2.3.3.2 Glucose is measured with colorimetric assays in automated analyzers.

2.3.3.3 Blood-CSF barrier damage can be expressed as albumin ratio $\left(\mathrm{Alb}_{\text {serum }} / \mathrm{Alb}_{\mathrm{CSF}}\right)$, or albumin quotient $\left(\mathrm{Alb}_{\mathrm{CSF}} / \mathrm{Alb}_{\text {serum }}\right) \times 10^{3}$ or $\times 10^{2}$ [35].

2.3.3.4 Degree of blood-CSF barrier damage can be semi-quantitatively expressed: "normal" for albumin quotient values up to $0.7 \%$; "mild," up to $2.0 \%$; and "moderate," up to $5.0 \%$; "severe," $>5.0 \%[36]$.

2.3.3.5 To reduce imprecision, albumin and $\operatorname{IgG}$ in serum and CSF should be measured with the same method and on the same analytical run.

2.3.3.6 Intrathecal IgG synthesis should be calculated with non-linear functions, such as Reiber formula [11], which take into account the bloodCSF barrier damage. IgG Index/Link index can yield false positive results in the presence of moderate/severe blood-CSF barrier damage [36].

2.3.3.7 Allowed methods for albumin and IgG measurement are as follows: nephelometry, turbidimetry, and radial immunodiffusion.

\subsubsection{Other tests}

2.3.4.1 CSF lactate determination is optional (useful in suspected septic meningitis/meningoencephalitis, when antibiotic therapy performed before the lumbar puncture could have normalized CSF glucose/serum glucose ratio). Due to lactate hydrophobicity, CSF lactate concentrations are independent from serum levels.

2.3.4.2 CSF IgA and IgM determinations are optional (no specific indications).

2.3.5 Calculation of antigen-specific antibody intrathecal synthesis

2.3.5.1 Starting from 3 to 4 weeks after onset of viral meningoencephalitis formulas for virusspecific antibody intrathecal synthesis can be positive and diagnostically useful [37]. In neuroborreliosis and West Nile virus, encephalitis antibody determinations are diagnostically mandatory, since PCRs are often negative $[38,39]$.

2.3.5.2 In suspected MS, testing for anti-measlerubella-varicella antibodies in serum and CSF (MRZ-reaction) should be restricted to atypical cases [40].

2.3.5.3 Tests for antigen-specific antibodies should be preferably performed in specialized laboratories of Centers of Infectious Diseases.

2.3.5.4 Antigen-specific antibody intrathecal synthesis can be calculated as follows [41]:

i) Determine total $\operatorname{IgG}$ concentration in serum and CSF.

ii) Dilute serum samples so that serum total IgG concentration equalizes CSF total IgG concentration.

iii) Test serum and CSF samples on ELISA for antigen-specific $\mathrm{IgG}$; express results in optical density (OD); dilute and retest samples if absorbance values fall outside the linear parts of the standard curves.

iv) Antigen-specific antibody index: $\mathrm{OD}_{\mathrm{CSF}} / \mathrm{OD}_{\text {serum }}$ ratio $>1.5-2.0$ indicates antigen-specific antibody intrathecal synthesis (the cutoff takes into account imprecision in total IgG and antigen-specific IgG measurements).

2.3.5.5 Immuno-affinity capillary blotting can be used for detecting antigen-specific OCBs [42]. It is labor-intensive, requires large amounts of viral proteins/peptides/lysates, and results of limited usefulness in routine practice.

\subsection{Determination of oligoclonal IgG bands}

2.4.1 Agarose or polyacrylamide gels for IEF ( $\mathrm{pH}$ range, 3.0-10.0; standard size, intermediate size (midigels), and small size (mini-gels)), either homemade or commercial, are allowed. 
2.4.2 Automated and non-automated IEF apparatus are allowed.

2.4.3 Equal amounts of paired serum and CSF IgG should be loaded on the gel adjacent lanes.

2.4.4 Amounts of the loaded-onto-the-gel serum and CSF IgG vary on the basis of different sensitivities of the staining methods (CSF sample concentration not allowed).

2.4.5 Refer to published protocols for blotting and IgGspecific detecting procedures [43]. For semiautomated instruments, follow manufacturer's instructions.

2.4.6 Sensitive staining techniques for $\mathrm{IgG}$ immunodetection are mandatory (e. g., peroxidase, avidin-biotin, chemiluminescence).

2.4.7 $\mathrm{pH}$ standards are useful for IEF set up.

2.4.8 High voltages require efficient IEF plate cooling systems. Stop IEF runs when low values of amperage are stable for $10 \mathrm{~min}$. Early stopping can cause partial OCB focalization, whereas excessively protracting runs OCB distortions.

2.4.9 Agarose gels: regularly dry the cathodal area, where electroendosmosis phenomena yield water, to prevent lack of OCB focalization and OCB distortions.

2.5 Interpretation of oligoclonal IgG band patterns

2.5.1 IEF interpretation, not requiring densitometers (human eyes are better in contrast discrimination), should be performed by two experienced operators. Particular attention should be paid to artifactual bands produced by the lack $\mathrm{pH}$ gradient homogeneity, due to regional weakness in conductivity within the gel; OCB-negative controls allow the recognition of such bands.

2.5.2 Adopt the following pattern classification: [1]

i) Type 1, diffuse polyclonal IgG distribution in both CSF and serum (absence of intrathecal IgG synthesis, normal pattern).

ii) Type 2, unique-to-CSF OCBs (presence of intrathecal IgG synthesis, typical of subacute/chronic CNS immunoinflammatory processes with immune response intrathecal compartmentalization).

iii) Type 3 , the so-called mixed pattern, uniqueto-CSF OCBs in addition to OCBs equal in serum and CSF (presence of intrathecal IgG synthesis, typical of acute/subacute CNS immuno-inflammatory processes with immune response persistence in the systemic compartment).

iv) Type 4, the so-called mirror pattern, OCBs equal in serum and CSF (absence of intrathecal IgG synthesis, typical of systemic immuno-inflammatory processes, with or without CNS involvement, and with systemic production of OCBs).

v) Type 5, the paraprotein pattern, OCBs equal in serum and CSF similarly to pattern 4 , but with regular and periodic spacing and decreasing intensity (absence of intrathecal IgG synthesis, presence of monoclonal gammopathy).

2.5.3 A positive OCB test is defined by the presence of at least two ("oligo") bands.

2.5.4 Not considered in the 1994 consensus [1], single CSF bands, with or without bands equal in serum and CSF can be detected and should be reported. They might associate with MS [44-46]. Follow-up CSF analysis is advisable.

2.5.5 Given the limits of human eyes, the interpretation of "faint bands" cannot be standardized. Retesting increased amounts of IgG (although it entails increases of polyclonal IgG background too), and follow-up CSF analysis is advisable.

2.5.6 Moderate/severe blood-CSF barrier damage or blood-contaminated CSF samples can reduce the chance of detecting OCBs, due to the increased amount of blood-derived polyclonal IgG (for being detectable, OCBs have to emerge from polyclonal IgG backgrounds).

2.5.7 Not considered in the 1994 consensus [1], OCBs equal in serum and CSF, but more intense in the CSF might indicate intrathecal IgG synthesis, as equal amounts of serum and CSF IgG are compared. However, imprecision can affect IgG measurements and sample dilutions, thus making unreliable the comparison of different intensity of CSF OCBs vs the serum counterparts. Follow-up CSF analysis is advisable.

2.5.8 Inter-laboratory reproducibility for OCBs is low $[2,47]$, thus limiting multicenter studies.

\subsection{Quality control and specimen storage}

\subsection{Biochemistry and proteins}

3.1.1 Internal controls should be included (e.g., pooled CSF samples).

3.1.2 External quality controls: follow the rules for laboratory accreditation.

3.2 Isoelectric focusing

3.2.1 Include positive (e.g., serum with monoclonal $\mathrm{IgG})$ and negative controls. 
3.2.2 External quality controls for OCBs should be planned at least yearly (e.g., AINI external quality control schemes).

3.2.3 Serum and CSF samples should be stored in aliquots at $-20{ }^{\circ} \mathrm{C}$, preferably at $-80{ }^{\circ} \mathrm{C}$.

\subsection{Report}

4.1. The following information should be reported:

4.1.1 Date and hour of lumbar puncture.

4.1.2 Anatomical site of origin of the CSF sample (lumbar, ventricular, cystic).

4.1.3 Test tube on which the analysis is performed (e.g., unique, third).

4.1.4 Appearance and color of the CSF before and after centrifugation.

4.1.5 Methods used for biochemico-immunological determinations.

4.1.6 Type of chamber for CSF cell counting/flow cytofluorimeter.

4.1.7 Reference values for each item.

4.1.8 Morphological description of CSF and IEF interpretation.

4.1.9 Comments (optional).

4.2 The reference values of CSF parameters should be assessed in appropriate control groups (diseased patients and healthy controls) for each laboratory. Reference values from published data can be adopted for both adult and pediatric patients. In adults with age $>60$ years, the physiological decrease of the CSF flow causes increases in albumin quotient (AQ) values, which can be thus non-indicative of blood-CSF barrier damage. For a more precise definition of the $\mathrm{AQ}$ upper reference limit, the formula 'AQ = age $/ 25+8$ ' has been proposed [48].

4.3 OCBs might be semi-quantitatively evaluated as follows: (a) single band; (b) two bands; (c) some bands $(n=3-6)$; (d) numerous bands $(n>6)$.

4.4 Reports may contain the sentence: "This laboratory follows procedures and methods standardized on behalf of the Italian Association of Neuroimmunology (date of the latest revision) and participates in the external quality control schemes promoted by the Association.”

\section{References}

1. Andersson M, Alvarez-Cermeno J, Bernardi G, Cogato I, Fredman P, Frederiksen J et al (1994) Cerebrospinal fluid in the diagnosis of multiple sclerosis: a consensus report. J Neurol Neurosurg Psychiatry 57:897-902

2. Sellebjerg F, Christiansen M (1996) Qualitative assessment of intrathecal IgG synthesis by isoelectric focusing and immunodetection: interlaboratory reproducibility and interobserver agreement. Scand J Clin Lab Invest 56:135-143
3. Nespolo A, Bernardi G (1995) Lo studio delle proteine liquorali: aspetti fisiopatologici e nuove prospettive diagnostiche. Corso CEFAR 1995: Le proteine dal laboratorio alla clinica

4. Reiber H (1995) External quality assessment in clinical neurochemistry: survey of analysis for cerebrospinal fluid (CSF) proteins based on CSF/serum quotients. Clin Chem 41:256-263

5. Teunissen CE, Malekzadeh A, Leurs C, Bridel C, Killestein J (2015) Body fluid biomarkers for multiple sclerosis - the long road to clinical application. Nat Rev Neurol 11:585-596

6. Granerod J, Ambrose HE, Davies NW, Clewley JP, Walsh AL, Morgan D et al (2010) Causes of encephalitis and differences in their clinical presentations in England: a multicentre, populationbased prospective study. Lancet Infect Dis 10:835-844

7. Gable MS, Sheriff H, Dalmau J, Tilley DH, Glaser CA (2012) The frequency of autoimmune N-methyl-D-aspartate receptor encephalitis surpasses that of individual viral etiologies in young individuals enrolled in the California Encephalitis Project. Clin Infect Dis 54: 899-904

8. Gresa-Arribas N, Titulaer MJ, Torrents A, Aguilar E, McCracken L, Leypoldt $\mathrm{F}$ et al (2014) Antibody titres at diagnosis and during follow-up of anti-NMDA receptor encephalitis: a retrospective study. Lancet Neurol 13:167-177

9. Freedman MS, Thompson EJ, Deisenhammer F, Giovannoni G, Grimsley G, Keir G et al (2005) Recommended standard of cerebrospinal fluid analysis in the diagnosis of multiple sclerosis: a consensus statement. Arch Neurol 62:865-870

10. Polman CH, Reingold SC, Banwell B, Clanet M, Cohen JA, Filippi $M$ et al (2011) Diagnostic criteria for multiple sclerosis: 2010 revisions to the McDonald criteria. Ann Neurol 69:292-302

11. Reiber H, Felgenhauer K (1987) Protein transfer at the blood cerebrospinal fluid barrier and the quantitation of the humoral immune response within the central nervous system. Clin Chim Acta 163: 319-328

12. Hutchinson M (2013) CSF oligoclonal bands are important in the diagnosis of multiple sclerosis, unreasonably downplayed by the McDonald criteria 2010: commentary. Mult Scler 19:719-720

13. Masjuan J, Alvarez-Cermeno JC, Garcia-Barragan N, DiazSanchez M, Espino M, Sadaba MC et al (2006) Clinically isolated syndromes: a new oligoclonal band test accurately predicts conversion to MS. Neurology 66:576-578

14. Zipoli V, Hakiki B, Portaccio E, Lolli F, Siracusa G, Giannini M et al (2009) The contribution of cerebrospinal fluid oligoclonal bands to the early diagnosis of multiple sclerosis. Mult Scler 15: $472-478$

15. Milo R, Miller A (2014) Revised diagnostic criteria of multiple sclerosis. Autoimmun Rev 13:518-524

16. Joseph FG, Hirst CL, Pickersgill TP, Ben-Shlomo Y, Robertson NP, Scolding NJ (2009) CSF oligoclonal band status informs prognosis in multiple sclerosis: a case control study of 100 patients. J Neurol Neurosurg Psychiatry 80:292-296

17. Siritho S, Freedman MS (2009) The prognostic significance of cerebrospinal fluid in multiple sclerosis. J Neurol Sci 279:21-25

18. Thangarajh M, Gomez-Rial J, Hedstrom AK, Hillert J, AlvarezCermeno JC, Masterman Tet al (2008) Lipid-specific immunoglobulin $\mathrm{M}$ in CSF predicts adverse long-term outcome in multiple sclerosis. Mult Scler 14:1208-1213

19. Garcia-Barragan N, Villar LM, Espino M, Sadaba MC, GonzalezPorque P, Alvarez-Cermeno JC (2009) Multiple sclerosis patients with anti-lipid oligoclonal IgM show early favourable response to immunomodulatory treatment. Eur J Neurol 16:380-385

20. Villar LM, Costa-Frossard L, Masterman T, Fernandez O, Montalban X, Casanova B et al (2015) Lipid-specific immunoglobulin $\mathrm{M}$ bands in cerebrospinal fluid are associated with a reduced risk of developing progressive multifocal leukoencephalopathy during treatment with natalizumab. Ann Neurol 77:447-457 
21. Mandrioli J, Sola P, Bedin R, Gambini M, Merelli E (2008) A multifactorial prognostic index in multiple sclerosis. Cerebrospinal fluid IgM oligoclonal bands and clinical features to predict the evolution of the disease. J Neurol 255:1023-1031

22. Gastaldi M, Zardini E, Franciotta D (2017) An update on the use of cerebrospinal fluid analysis as a diagnostic tool in multiple sclerosis. Expert Rev Mol Diagn 17:31-46

23. Majed M, Fryer JP, McKeon A, Lennon VA, Pittock SJ (2016) Clinical utility of testing AQP4-IgG in CSF: guidance for physicians. Neurol Neuroimmunol Neuroinflamm 3:e231

24. Wingerchuk D, Banwell B, Bennett J, Cabre P, Carroll W, Chitnis T et al (2015) International consensus diagnostic criteria for neuromyelitis optica spectrum disorders. Neurology 85:177-189

25. Stoeck K, Sanchez-Juan P, Gawinecka J, Green A, Ladogana A, Pocchiari M et al (2012) Cerebrospinal fluid biomarker supported diagnosis of Creutzfeldt-Jakob disease and rapid dementias: a longitudinal multicentre study over 10 years. Brain 135:3051-3061

26. van Harten AC, Kester MI, Visser PJ, Blankenstein MA, Pijnenburg YA, van der Flier WM et al (2011) Tau and p-tau as CSF biomarkers in dementia: a meta-analysis. Clin Chem Lab Med 49:353-366

27. Molinuevo JL, Blennow K, Dubois B, Engelborghs S, Lewczuk P, Perret-Liaudet A et al (2014) The clinical use of cerebrospinal fluid biomarker testing for Alzheimer's disease diagnosis: a consensus paper from the Alzheimer's Biomarkers Standardization Initiative. Alzheimers Dement 10:808-817

28. Steinacker P, Feneberg E, Weishaupt J, Brettschneider J, Tumani H, Andersen PM et al (2016) Neurofilaments in the diagnosis of motoneuron diseases: a prospective study on 455 patients. J Neurol Neurosurg Psychiatry 87:12-20

29. Fokke C, van den Berg B, Drenthen J, Walgaard C, van Doorn PA, Jacobs BC (2014) Diagnosis of Guillain-Barré syndrome and validation of Brighton criteria. Brain 137:33-43

30. Joint Task Force of the EFNS and the PNS (2010) European Federation of Neurological Societies/Peripheral Nerve Society guideline on management of chronic inflammatory demyelinating polyradiculoneuropathy: report of a joint task force of the European Federation of Neurological Societies and the Peripheral Nerve Society-first revision. J Peripher Nerv Syst 15:1-9

31. Martino G, Grimaldi LM, Moiola L, Filippi M, Martinelli V, Comi $\mathrm{G}$ et al (1990) Discontinuous distribution of IgG oligoclonal bands in cerebrospinal fluid from multiple sclerosis patients. J Neuroimmunol 30:129-134

32. Bertolotto A, Malentacchi M, Capobianco M, di Sapio A, Malucchi S, Motuzova Y et al (2016) The use of the 25 Sprotte needle markedly reduces post-dural puncture headache in routine neurological practice. Cephalalgia 36:131-138

33. Cruickshank AM (2001) ACP best practice no 166: CSF spectrophotometry in the diagnosis of subarachnoid haemorrhage. J Clin Pathol 54:827-830
34. Steele RW, Marmer DJ, O'Brien MD, Tyson ST, Steele CR (1986) Leukocyte survival in cerebrospinal fluid. J Clin Microbiol 23: 965-966

35. Tibbling G, Link H, Ohman S (1977) Principles of albumin and IgG analyses in neurological disorders. I. Establishment of reference values. Scand J Clin Lab Invest 37:385-390

36. Thompson E (1988) The CSF proteins: a biochemical approach. Elsevier, Amsterdam

37. Gregoire SM, van Pesch V, Goffette S, Peeters A, Sindic CJ (2006) Polymerase chain reaction analysis and oligoclonal antibody in the cerebrospinal fluid from 34 patients with varicella-zoster virus infection of the nervous system. J Neurol Neurosurg Psychiatry 77: 938-942

38. Koedel U, Fingerle V, Pfister HW (2015) Lyme neuroborreliosisepidemiology, diagnosis and management. Nat Rev Neurol 11:446456

39. Davis LE, DeBiasi R, Goade DE, Haaland KY, Harrington JA, Harnar JB et al (2006) West Nile virus neuroinvasive disease. Ann Neurol 60:286-300

40. Hottenrott T, Dersch R, Berger B, Rauer S, Eckenweiler M, Huzly $\mathrm{D}$ et al (2015) The intrathecal, polyspecific antiviral immune response in neurosarcoidosis, acute disseminated encephalomyelitis and autoimmune encephalitis compared to multiple sclerosis in a tertiary hospital cohort. Fluids Barriers CNS 12:27

41. Reiber H, Lange P (1991) Quantification of virus-specific antibodies in cerebrospinal fluid and serum: sensitive and specific detection of antibody synthesis in brain. Clin Chem 37:1153-1160

42. Dorries R, Ter Meulen V (1984) Detection and identification of virus-specific, oligoclonal IgG in unconcentrated cerebrospinal fluid by immunoblot technique. J Neuroimmunol 7:77-89

43. Olsson T, Kostulas V, Link H (1984) Improved detection of oligoclonal $\mathrm{IgG}$ in cerebrospinal fluid by isoelectric focusing in agarose, double-antibody peroxidase labeling, and avidin-biotin amplification. Clin Chem 30:1246-1249

44. Davies G, Keir G, Thompson EJ, Giovannoni G (2003) The clinical significance of an intrathecal monoclonal immunoglobulin band: a follow-up study. Neurology 60:1163-1166

45. Franciotta D, Bergamaschi R, Amato MP, Zardini E, Persico A, Portaccio E et al (2005) Clinical correlations of CSF single IgG bands. J Neurol 252:1274-1275

46. Ferraro D, Franciotta D, Bedin R, Solaro C, Cocco E, Santangelo M et al (2017) A multicenter study on the diagnostic significance of a single cerebrospinal fluid IgG band. J Neurol 264:973-978

47. Franciotta D, Avolio C, Lolli F (2005) Between-laboratory variability in oligoclonal IgG band numbering. Clin Chem 51:270-272

48. Hegen H, Auer M, Zeileis A, Deisenhammer F (2016) Upper reference limits for cerebrospinal fluid total protein and albumin quotient based on a large cohort of control patients: implications for increased clinical specificity. Clin Chem Lab Med 54:285-292 